\title{
RECENT RESULTS AND PERSPECTIVES AT CDF AND DØ
}

\author{
H.E. MONTGOMERY \\ Fermi National Accelerator Laboratory \\ P.O. Box 500 \\ Batavia, IL60510 U.S.A.
}

\begin{abstract}
.
Over the course of the past years the experimental measurements performed by the two large collaborations, CDF and DØ, at the Fermilab Tevatron Collider have fueled advances in our understanding of physics at the energy frontier. At the present time the accelerator complex and the two detectors are undergoing substantial improvements. In this paper, we provide a discussion of some recent results which in turn provides a framework within which we can look to future prospects.
\end{abstract}

\section{Introduction}

The Tevatron with the aid of its associated detectors, CDF and D $\varnothing$, has made its most significant mark on experimental particle physics progress with the observation of the top quark. However this was only one of a number of important contributions[1,2]. In this paper we describe some examples of recent results[3] and look forward to future running of the experiments with significantly increased luminosity. The latter is possible as a result of the introduction of a new accelerator, The Main Injector, into the Fermilab complex; this is described in Section 2. Both detectors are undergoing upgrades which will enable them to operate in the new environment with greatly enhanced capabilities. These changes are briefly described in Section 3. In Section 4, we discuss the physics accessible at the $p \bar{p}$ collider working our way through the physics of the strong interaction, QCD, to the physics beyond the standard model. At present much of the latter remains speculative but provides the framework for the future experimental work. Finally we offer a brief conclusion in Section 5. 


\section{The Tevatron and Main Injector}

The Tevatron is a $p \bar{p}$ collider[4]. The energy is $900 \mathrm{GeV}$ in each beam and it is the highest energy collider in the world. In a hadron collider, the effective parton-parton energy is controlled not only by the machine energy but also by the luminosity. At high luminosity the rate of higher momentum scatters increases. Thus, while $t \bar{t}$ production was accessible kinematically from the first day of the collider operation, it was not until ten years later that sufficient high momentum scatters had been accumulated to make the $t \bar{t}$ production observable experimentally.

The luminosity of the machine is, to a good approximation, controlled by the total number of antiprotons available. In turn this depends on the production rate and the cooling rate. The new Main Injector, a rapid cycling proton synchrotron operating at $150 \mathrm{GeV}$, will be used for antiproton production. An innovation is the introduction of the Recycler, an $8 \mathrm{GeV}$ storage ring constructed with permanent magnets. At the end of each Tevatron store, approximately half of the initial antiprotons are still present. Most of the luminosity degradation is a result of beam blow-up. The antiprotons are to be decelerated in the Tevatron, then in the Main Injector to $8 \mathrm{GeV}$, and stored and recooled in the Recycler. After cooling the the anti-proton capacity of the complex is increased by a factor of two. With these measures, the instantaneous luminosity in the collider is anticipated to increase to about $2 \times 10^{32} \mathrm{~cm}^{-2} \mathrm{~s}^{-1}$. This will make an integrated luminosity of about $4 \mathrm{fb}^{-1}$ available in the next few years perhaps rising to $10-30 \mathrm{fb}^{-1}$ over the next six to seven years. This is to be compared with the $0.1 \mathrm{fb}^{-1}$ of the present data set. The Main Injector has been comissioned and the Tevatron is operational for fixed target physics. At the same time the energy per beam will be increased from $900 \mathrm{GeV}$ to close to $1000 \mathrm{GeV}$. For high mass processes, such as top production the cross section increases by $30-40 \%$.

\section{CDF and DØ Detectors}

The CDF detector, see Fig. 1, has been operational for more than ten years. It contains a large solenoid which provides a 1.4 Tesla magnetic field in the tracking volume. That volume is surrounded by a scintillator/lead/iron calorimeter and a non-magnetic muon detector. The current upgrade concentrates on completely replacing the tracking detectors. The outer tracking will be provided by a large open cell drift chamber with shorter drift distances than the previous detector. At inner radii, this is complemented by a comprehensive silicon detector system both to enhance the tracking capability and to provide detection of secondary decay vertices. The detection of $B$ hadrons, both for their own sake and as indicators of the decays of higher 


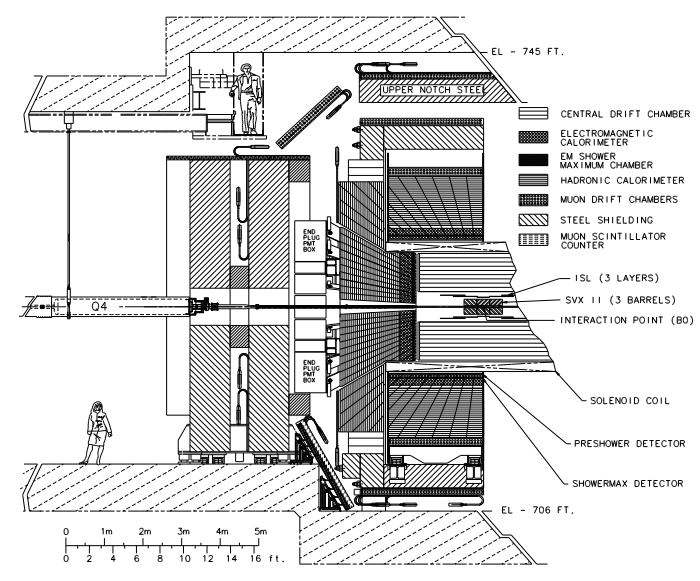

Figure 1. The upgraded CDF detector.

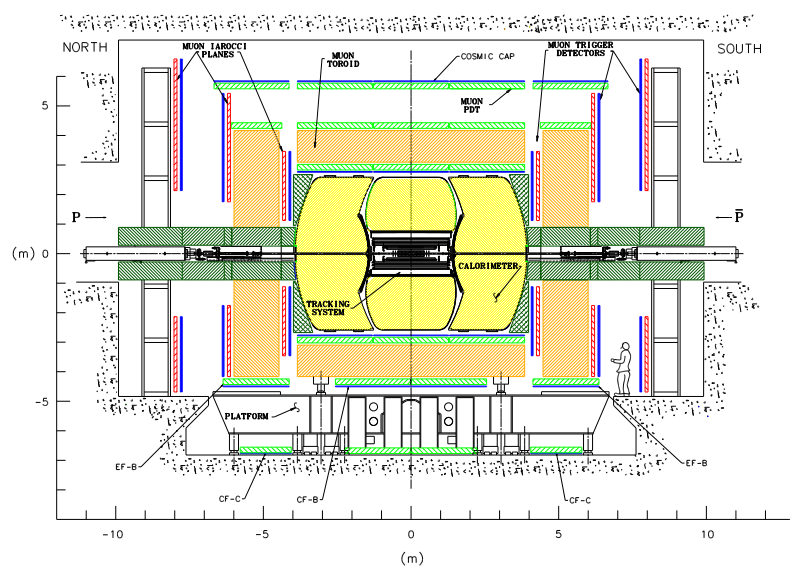

Figure 2. The upgraded D $\varnothing$ apparatus.

mass states, top and perhaps the Higgs particle, places a strong premium on this capability.

The D $\varnothing$ detector, see Fig. 2, is characterised by a three-cryostat liquid Argon/Uranium calorimeter with good electron and jet resolutions. The muon system consists of detectors inside and outside of large iron toroids in both central and forward regions. The forward muon system is being equipped with new trigger and tracking detectors to accomodate the upgraded accelerator parameters. A new superconducting solenoid has been installed in the tracking volume and the particle detection will be performed using a scintillating fiber tracker and a 800,000 channel silicon tracker.

Initially the collider will operate with 496 nsec between collisions of 
the bunches but this will eventually be reduced to 132 nsec. Pipelines, analogue in some cases, digital in others, have been introduced in the front end electronics in both of the new detectors. The data acquisition systems have also been upgraded to accomodate event rates of several tens of $\mathrm{Hz}$, to accomodate the overall luminosity increase. The detectors are to be operational in early 2001.

\section{Physics}

\subsection{QCD}

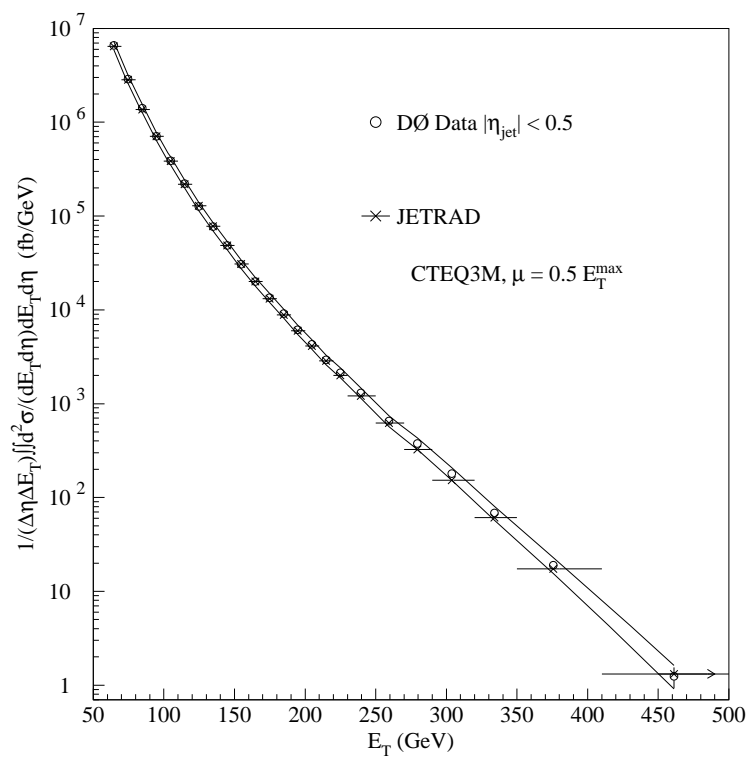

Figure 3. DØ inclusive jets cross section and a comparison with a next-to-leading order QCD prediction.

Production cross sections in $p \bar{p}$ collisions are calculated by convoluting the parton distribution functions in proton and anti-proton, respectively, with the appropriate hard parton-parton scattering cross section[5]. The parton distribution functions are derived from a number of measurements, primarily from lepton scattering, and are evolved to the appropriate hard scattering scale. That this paradigm works well is demonstrated in Fig. 3 where we see agreement of the calculations with the inclusive jet production cross sections from $\mathrm{D} \varnothing$ over many orders of magnitude. Deviations from perfection would be signs of either changes required to the parton distribution functions or, perhaps, a sign of physics beyond the paradigm. While there have been alarms, the currently accepted view is that agree- 
ment between predictions and data is good[6]. A corollary is that from these results, from measurements of the dijet mass distributions, and from measurements of the angular distributions, a lower limit on the scale of any possible compositeness can be set at about 2-3 TeV.

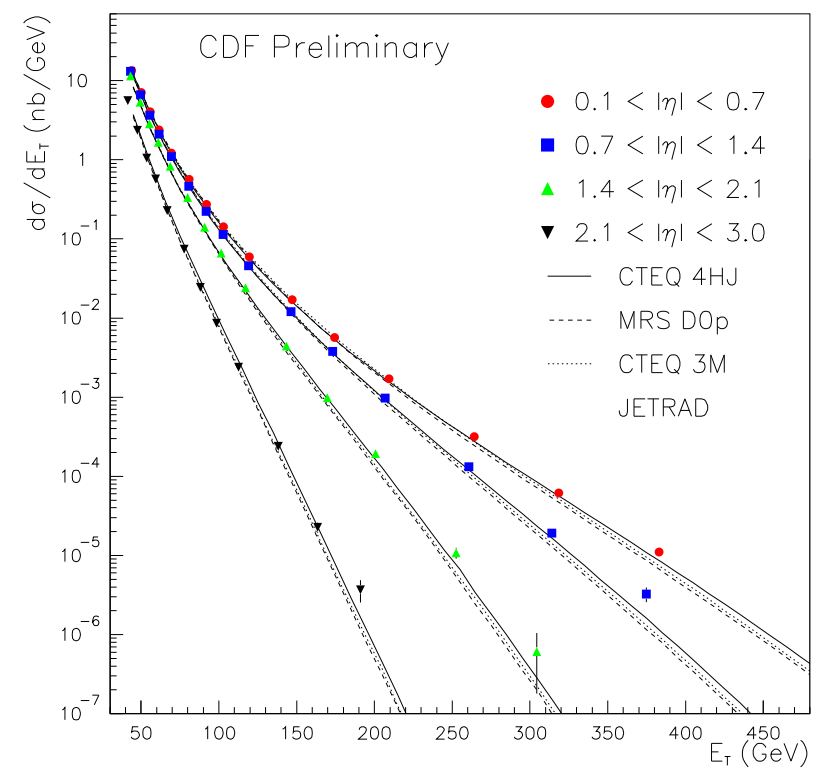

Figure 4. CDF Inclusive Jets at large rapidity.

The prescription described above should also permit an incisive comparison between cross sections measured at different energies, $1800 \mathrm{GeV}$ and $630 \mathrm{GeV}$, appropriately scaled in transverse momentum. This comparison should be fairly insensitive to the choice of parton distribution functions; however what is found is that the theory differs from the preliminary measurement $[6,7]$ by about a factor of two. Agreement can only be achieved by modifying the choices of renormalisation and factorisation scales. Recently the measurements have been extended[8] in rapidity. Again these measurements are sensitive to different aspects of the parton distribution functions. In this case, as can be seen in Fig. 4, agreement is good.

Traditionally, experiments at hadron colliders have used a cone algorithm[6] to reconstruct jets. In contrast, work using electron-positron, or lepton-nucleon collisions has employed algorithms which construct jets from elementary objects such as the individual charged tracks or the energy deposited in a calorimeter by a single hadron. Such algorithms[9] are known 


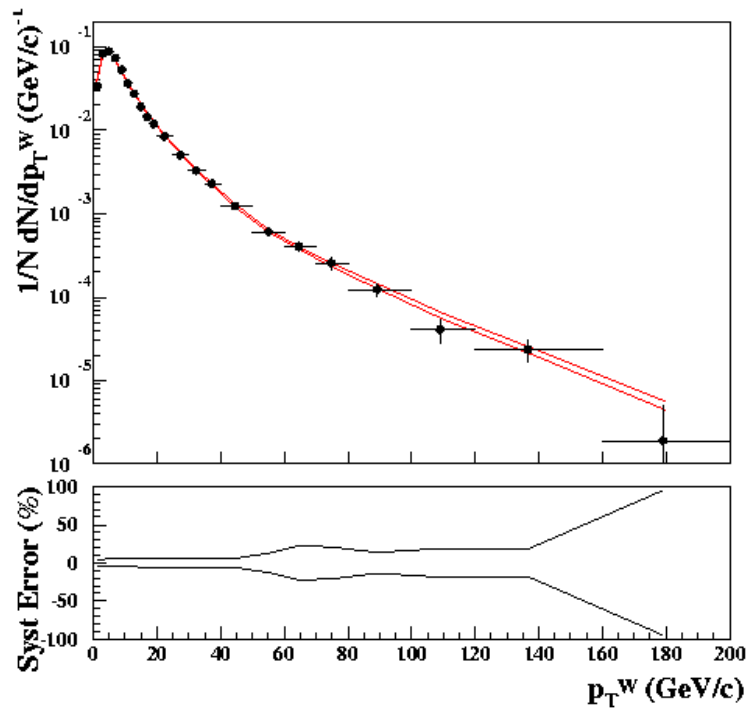

Figure 5. $W$-boson $p_{T}$ spectrum as measured by $\mathrm{D} \varnothing$, the curves are the bounds of the smeared next-to-leading order predictions.

as $k_{T}$, Jade, or Durham algorithms. Recently we have started to use them in $\bar{p} p$ experiments to look at the jet substructure. $\mathrm{D} \varnothing$ has a preliminary result[10] which suggests that the multiplicity of sub-jets in gluon jets is larger than that in quark jets. In the future, this approach could well form the basis for distinguishing jet identities in other analyses such as Higgs searches.

When the production of $W$ and $Z$ bosons was first established at the CERN S $p p$ S there were fewer than ten each of these particles. The number of $W$ bosons observed by each of CDF and $\varnothing \varnothing$ now approaches one hundred thousand. These data samples have permitted the use of the weakly interacting final state particles in investigations of QCD, in a manner analogous to the use of virtual bosons in the initial state in neutrino experiments.

$W$ and $Z$ production is dominated by the lowest order parton model annihilation of valence quarks from proton and antiproton. The QCD calculations agree very well with the cross sections which have been measured $[11,12]$ with a precision of a few $\%$. The higher order QCD corrections introduce transverse momentum for the bosons and at high $p_{T}$, the recoiling hadronic system may contain one or more jets. The transverse 


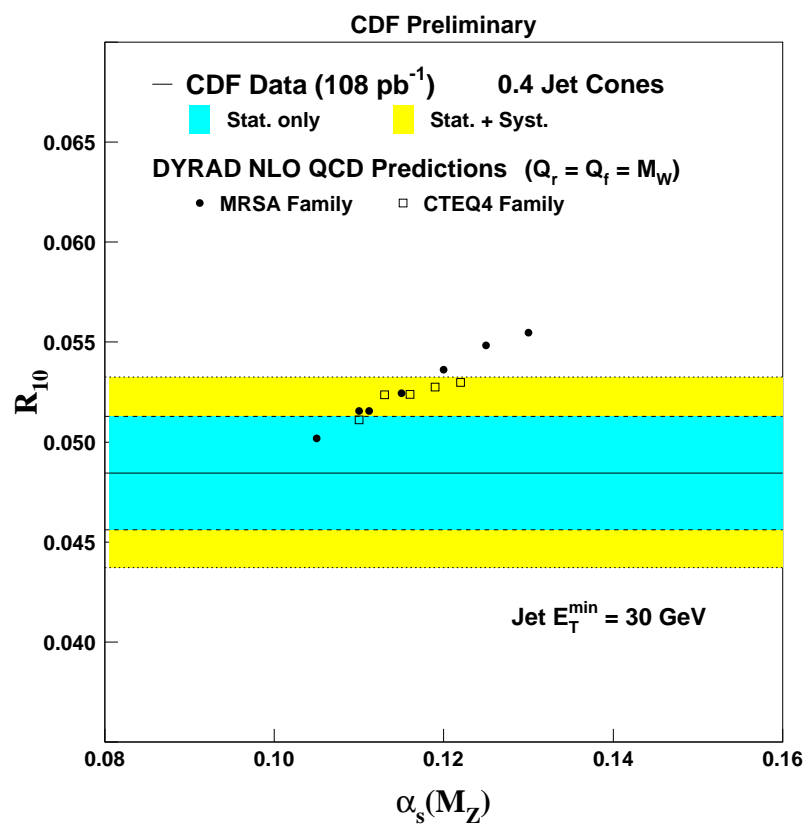

Figure 6. Fraction of $W$-boson events with at least one jet as measured by CDF. The dependence of the prediction on the strong interaction coupling is also shown.

momentum spectra at high momenta are expected to be well described by perturbative calculations while at low $p_{T}$ non-perturbative effects are expected. Resummation of some of the logarithms is expected to be necessary. Recent results on both $Z$ and $W$ spectra[12,13] are surprisingly well described by the extant predictions. This is illustrated for $W$ production in Fig. 5.

The CDF measurement[14] of the fraction of $W$ production containing one or more jets above a given threshold, $\mathrm{R}_{10}$, is shown in Fig. 6. Conceptually, this is a classic measurement of the strength of the QCD coupling strength. The events with at least a single jet contain at least one strong interaction vertex, the total production is dominated by events with no such vertex. As usual, since $\alpha_{S}$ is not so small, there are higher order corrections. Nevertheless, the theory at next to leading order provides an adequate description of the results.

\subsection{FLAVOR PHYSICS}

Thus far, three generations of quarks have been observed; each generation contains an up-type quark and a down-type quark. The weak states are 
mixtures of the eigenstates of the strong interaction. This mixing[15], is described by a $3 \times 3$ matrix of transition amplitudes between the quark states. The Cabibbo-Kobayashi-Maskawa matrix can be described by four parameters. One of these is a phase through which the formalism accomodates and describes the $\mathrm{CP}$ violation observed in the kaon system. In turn the parameters of the matrix, assuming that there are only three generations, can be represented, as shown in Fig. 7, by a triangle. Many of the properties of this triangle are accessible by measurements of the kaon system, however measurements of the properties of $B$ hadrons are becoming increasingly important.

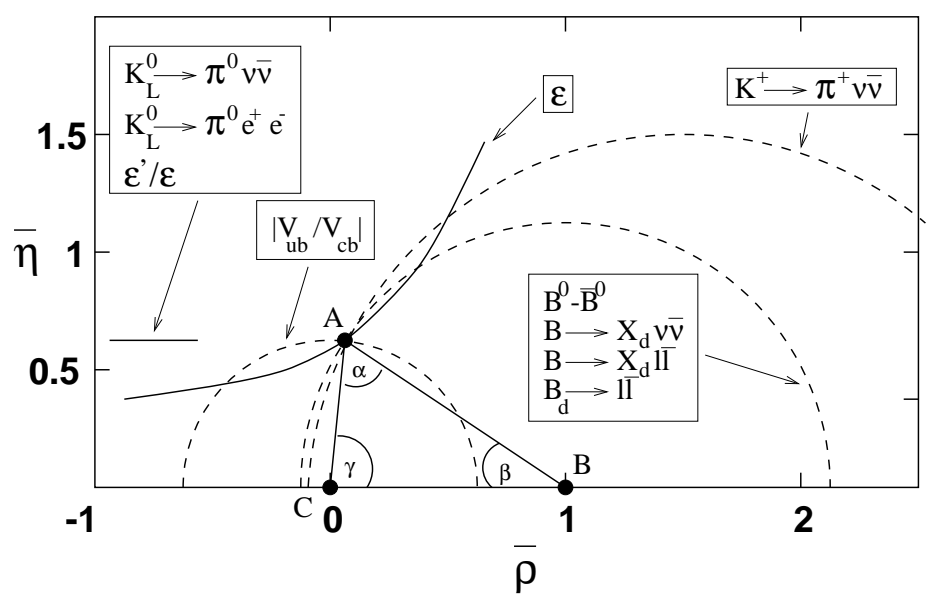

Figure 7. The Unitarity Triangle associated with the Cabbibo-Kobayashi-Maskawa flavor mixing matrix and the measurements possible in the kaon system.

On the scale of $1800 \mathrm{GeV}$, the mass of the $b$ quark is small; the production cross section is about $1 / 1000$ of the total cross section. Measurements of the cross section by both experiments in the central region are about a factor of two higher than expected. Production extends over about six units of rapidity and $\mathrm{D} \varnothing$ has made measurements[17] in the forward direction which are even higher, a factor four, with respect to the predictions.

As a result of the high energy, $B$ hadrons which contain a strange quark or a charm quark are produced in addition to those containing up and down quarks. For example, in 1998, CDF observed[18] the $B_{c}$. This state was observed in a semi-leptonic decay mode with a missing neutrino. Nevertheless, the signal was unequivocal and a good determination of the mass was made.

Using its silicon vertex detector, CDF has accumulated a set of measurements of the lifetimes of various $B$ hadrons. These results are displayed in Fig. 8. It is immediately clear that, unlike in the charm system, the lifetimes 


\section{CDF B Lifetimes}

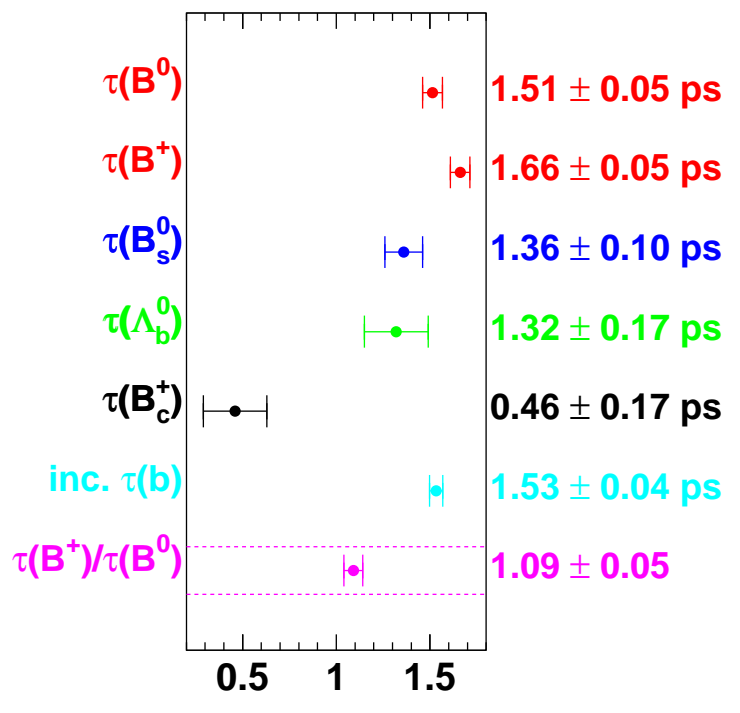

Figure 8. A compilation of B hadron lifetime measurements from CDF.

of charged and neutral $B$ mesons are rather similar. This is understood to be the result of the dominance of the simplest diagrams as a result of the high $b$-quark mass. We also notice that the lifetimes of both the $\Lambda_{b}$ baryon and of the $B_{s}$ meson are quite similar to that of the $B^{+}$and $B^{0}$. The lifetime of the $B_{c}$ is affected by the decay of the $c$ quark as well as that of the $b$ quark, hence the observed factor of two shorter lifetime[19].

The neutral $B$ mesons mix in a manner similar to the neutral kaons and the mixing for $B_{d}$ has been measured. Thus far the attempts to detect $B_{s}$ mixing have not met with success. The most recent measurements[20] from CDF place a limit $\left(\Delta m_{s}<5.8 \mathrm{ps}, x_{s}=\Delta m_{s} / \Gamma_{s}>7.9\right)$ as good as any achieved by the LEP experiments or by SLD at SLAC. Extrapolating to Run II, we expect sensitivities in the range $x_{s} \geq 25$ from each experiment, likely more from $\mathrm{CDF}$, thus comfortably covering the expected range.

The goal of flavor physics experiments is to measure all the parameters with sufficient detail to overconstrain the CKM matrix and, if possible, to break the model. Recently CDF presented results of their measurements of the $\mathrm{CP}$ violating asymmetry in the decay $B \rightarrow J / \psi K_{S}^{0}$ which determines $\sin 2 \beta$. They have one sample of events for which the proper decay time is measured and one for which only the time-integrated measurement is obtained. The results are displayed in Fig. 9. They find[21] $\sin 2 \beta=0.79_{-0.44}^{+0.41}$ 


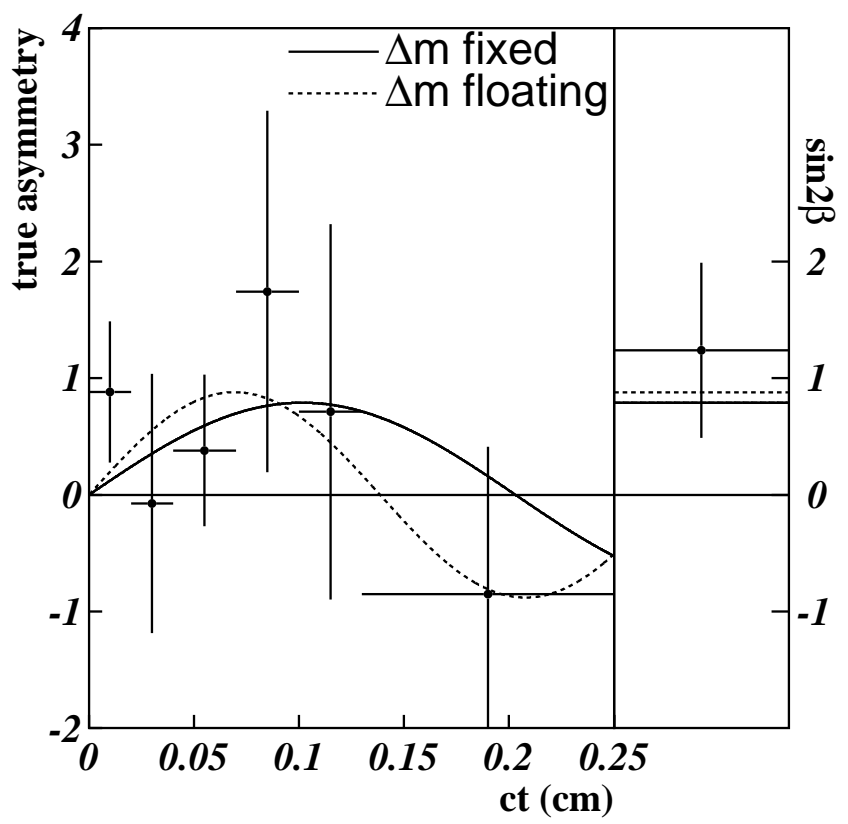

Figure 9. Measurement of the CP violating Asymmetry in $J / \psi K_{S}^{0}$ from CDF. On the left the asymmetry is shown as a function of lifetime, and on the right, for an independent sample, the time-integrated asymmetry is shown.

suggesting a positive value at about the $90 \%$ c.l. A feature of this measurement is the use of several different flavor tagging techniques. With approximately $2 \mathrm{fb}^{-1}$ and the upgraded detectors, the uncertainty on $\sin 2 \beta$ will be reduced below 0.1 for each experiment. Similar uncertainties are projected for $\sin 2 \alpha$ although the interpretation for this case is considered to be more difficult. Measurement of the third angle, $\gamma$, will be a challenge.

The mass of the top quark will be considered as an electroweak parameter and discussed in the following section. As far as the determination of the couplings and other characteristics of the top quark are concerned, studies are in their infancy. The observed cross section certainly seems to be consistent with that expected. Further, the mix of events of different topologies as yet show little deviation from expectations. These facts limit somewhat the liberties which can be taken with the coupling $V_{t b}$ between top and bottom quarks and possible decays, for example into a charged Higgs boson plus a bottom quark.

One of the fascinating properties of the top quark is its short lifetime. This implies that the $W$ boson into which it is expected to decay will have a well defined polarisation. CDF has measured[22] that the fraction 
of longitudinally polarised $W$ bosons is $0.97 \pm 0.37$ (stat) \pm 0.12 (syst); the expected value is 0.70 . The short lifetime means that decays occur before hadronisation and as a result correlations between the spins of the top and anti-top quarks, generated by the annihilation production diagram, are expected to survive and manifest themselves in the relative spins of the observed states. D $\varnothing$ has made a measurement using the sample of events in which each of the $W$ bosons decayed into a lepton and neutrino. While statistics are small, this sample is relatively background free. Along with that of the $b$ quark, the angular distribution of the charged lepton is the most powerful indicator of this property. The measure of the correlation is the parameter $\kappa$ defined such that $-1<\kappa<+1$ with $\kappa=1$ expected in the standard model. D $\varnothing$ observes[23] $\kappa>-0.25$ at $68 \% \mathrm{cl}$.

\subsection{ELECTROWEAK PHYSICS}

In an earlier section we mentioned the large numbers of $W$ and $Z$ bosons produced at the Tevatron. In addition to single bosons, boson pair production is also kinematically accessible. The various possible processes are sensitive to the triple boson couplings. These couplings among the gauge bosons are a fundamental feature of the non-Abelian electroweak theory and markedly different from the purely electromagnetic theory in which the gauge bosons, the photons, carry no charge. In the electroweak theory the non-Abelian couplings lead to cancellations among the different diagrams. For example, without them the production cross section for several diboson final states would diverge at high energy and would violate unitarity. As a result, searches for these rare processes have led to limits[24] on the possible deviations from these coupling strengths from their standard model values. As an indicator of what the future may hold, CDF has observed an excellent candidate for the pair production of $Z$ bosons and $\mathrm{D} \varnothing$ has an excellent candidate $W Z$ event.

The copious production of $W$ bosons permits a study of their properties. The ratio of production cross section times branching ratios for $W$ and $Z$ can be related, using measurements from LEP of the $Z$ boson properties, to the total $W$-boson width[11]. The standard model value for the $W$ leptonic width is used as input as well as the calculated ratio of the production cross sections. Alternatively the high-transverse-mass tail of the the $W$ boson event distribution can be used to directly measure[25] the width. At present the indirect technique is most precise; however, it does depend on standard model assumptions. The hope would be that in the future both measurements would be of sufficient precision to provide an additional constraint on the standard model and a determination of the leptonic width of the $W$ boson. 
The gauge sector of the standard electroweak model is specified by three quantities usually taken to be the muon weak decay constant, the electromagnetic fine-structure constant and the mass of the $Z$ boson. These three quantities unambiguously lead to a prediction of the mass of the $W$ boson at lowest order in the theory. Higher order corrections are expected from loop diagrams containing the fermions and hence dominated by the top quark, and a diagram with emission and reabsorbtion of the Higgs boson, if such exists. A precise measurement of the $W$ boson mass is therefore an important goal.

$W$ bosons are observed by detecting a charged lepton and measuring the hadronic recoil vector. This permits the neutrino kinematics to be inferred using momentum conservation, but only in the transverse plane. The mass of the boson is therefore deduced by fitting templates generated with a range of masses to one or all of the two lepton transverse momentum spectra and the transverse mass spectrum. (The transverse mass is a quantity constructed analogously to the effective mass but which is defined using only the transverse components of the energy-momentum vectors.) Each of these spectra is sensitive to different aspects of the measurement. The charged lepton recoils are very sensitive to the $W$ transverse momentum spectrum but largely independent of the hadron measurements. The neutrino recoil is very sensitive to both the boson transverse momentum and the hadronic measurement. The transverse mass is moderately sensitive to the lepton and hadronic measurements but relatively insensitive to the boson transverse momentum. Hence, use of all the measurement information provides powerful cross checks; maximum sensitivity is achieved by combining all measurements. These features, in addition to the use of the $Z$-boson data for in situ calibration, permit the experiments to match reduction in systematic uncertainties to reductions in statistical uncertainties as the samples increase.

The current results $80.433 \pm 0.079 \mathrm{GeV}$ from the CDF measurement[26] and $80.482 \pm 0.091 \mathrm{GeV}$ from the $\mathrm{D} \varnothing$ measurement[27] lead to a combined result of $80.448 \pm 0.062$. The CDF measurement uses both electrons and muons, primarily in the central regions of the detector. The DØ measurement is limited to electrons but exploits data from the end as well as the central calorimeters. These results are in excellent agreement with the combined results[28] of the four LEP experiments of $80.350 \pm 0.056 \mathrm{GeV}$. They also demand the inclusion of electroweak loop corrections to be compatible with the three basic electroweak parameters. The good behavior of systematic errors discussed above is illustrated in Fig. 10 which contains a projection of the evolution of the $W$ mass uncertainty as the integrated luminosity is increased even further with the upgraded detectors. One can note that this projection preceded the most recent results but that these 


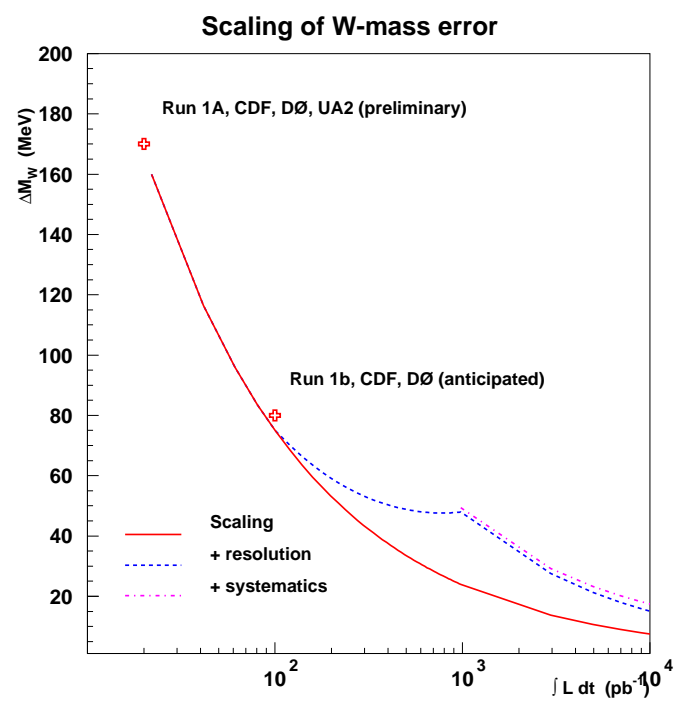

Figure 10. Expected evolution of the precision of a measurement of the $W$-boson mass at the Tevatron Collider.

$100 \mathrm{pb}^{-1}$ measurements fit well on the curve. The cusp occurs as the number of interactions per crossing increases followed by a further change in the time spacing between bunch crossings. It would seem that an uncertainty of $40 \mathrm{MeV}$ per experiment is not out of the question.

Because of its large mass, the top-quark is currently only directly accesible at the Tevatron. The current data samples have allowed CDF and D $\varnothing$ to measure[29] the mass. The techniques vary depending on the channel used. CDF uses final states in which the top and the antitop each decays into three jets, two light quark jets from the intermediate $W$ boson and a $b$ - or $\bar{b}$ - quark jet. However in this channel the background is large and the resulting measurement has an uncertainty of around $10 \mathrm{GeV}$. In the dilepton channel, even with two missing neutrinos, the mass can be determined; however, the low statisistics offset a rather good understanding of the systematic uncertainties. Again from each experiment the uncertainties are of order $10 \mathrm{GeV}$. For each experiment the dominant measurement comes from the "lepton-plus-jets" channel in which one of the intermediate $W$ bosons decays leptonically giving a charged lepton and a missing neutrino while the other decays into light quarks. The final state then contains four jets and a lepton for which each of the momentum vectors is fully measured, and a neutrino for which only the transverse components are measured. Using the mass constraints, those from the intermediate $W$ bosons and that from demanding that the top and antitop masses be the same, leads to kinematic 


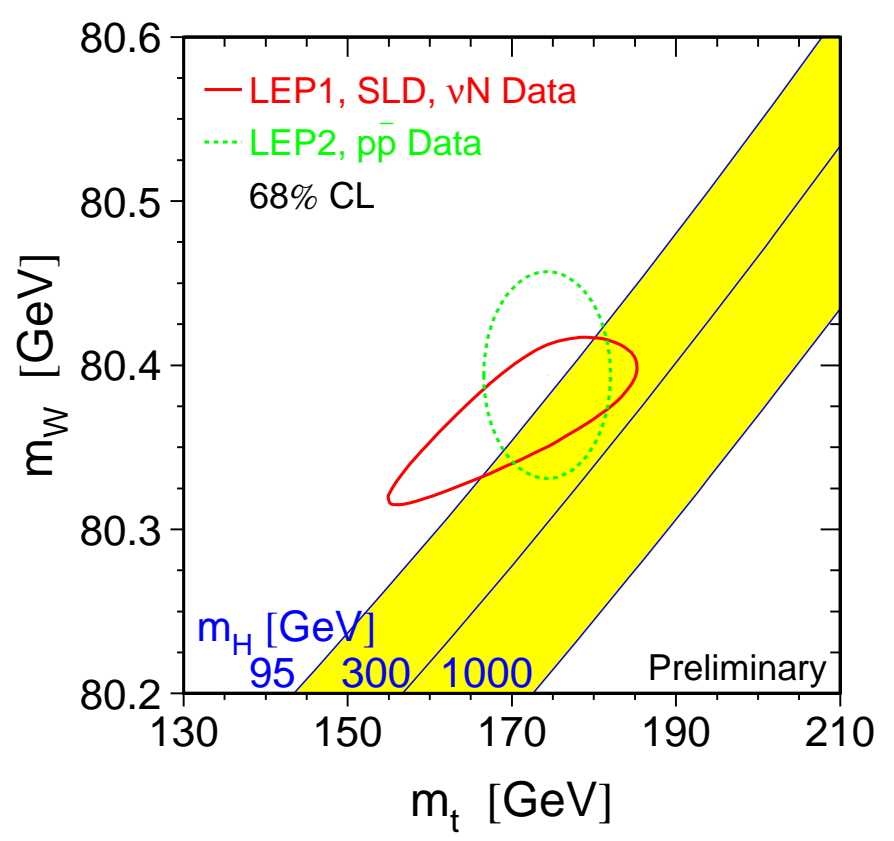

Figure 11. $M_{W}$ versus $m_{t}$ showing that a light Higgs is favoured by the current data.

fits with two constraints. Account has to be taken of the possible combinations. These may be restricted if the $b$-quark jet is well identified, either by a displaced decay vertex or from a soft charged lepton from the $b$-quark decay. Using this channel, $\mathrm{CDF}$ achieves an uncertainty of about $7 \mathrm{GeV}$ including systematics whereas that from $\mathrm{D} \varnothing$ is $8 \mathrm{GeV}$. All these measurements are combined taking into account the correlations in uncertainties, both those derived from common experimental errors (between channels in a given experiment, and those derived from common techniques between experiments. The result is that $m_{t}=174.3 \pm 3.2 \pm 4.0 \mathrm{GeV}$. This makes the mass of the top quark the best measured of all the quark masses.

We can then use the top mass and the $W$ mass and compare them with the basic electroweak predictions. As indicated earlier, the Higgs boson mass enters into the calculations of the electroweak loops. Hence the combination of all the electroweak measurements has sensitivity to the mass of the putative Higgs boson. This is illustrated in Fig. 11; we see that the data favour a light Higgs, of order 100-200 GeV. The uncertainties are rather large but this tendency in the existing data both from the Tevatron and 
from LEP/SLD has lent excitement to the searches current at LEP and to work on the upgrades of the Tevatron experiments.

The top mass determination described above is dominated by the uncertainties in the jet energy scale calibration. In future runs we expect that these uncertainties can be reduced using the data themselves. CDF has observed a $W$-mass peak in the decay jets from top and, in a $b \bar{b}$ data sample, has observed the peak from the $Z$ boson. Taking into account secondary vertex triggers, which each experiment expects to use in the upcoming running, the latter will provide a powerful jet calibration tool. As a result we can look forward to a reduction of the uncertainty on the mass of the top-quark to less than $2 \mathrm{GeV}$ from each of $\mathrm{CDF}$ and $\mathrm{D} \varnothing$ in the next few years.

\subsection{BEYOND THE STANDARD MODEL}

It is the duty of experimenters at the highest energy colliders to search for phenomena not previously observed. These searches are necessarily guided by how we imagine the underlying physics. At any given time, certain scenarios enjoy more popularity than others. We have seen in the past that states decaying into lepton pairs have often provided discoveries, for example the $J / \psi$ and the $Z$ boson. QCD breaks the electroweak symmetry, however not with sufficient strength to explain the masses of the $W$ and $Z$, so some have postulated an analogous interaction, technicolor, to generate such masses. At the present time, many theorists believe that supersymmetry should play a role and the phenomenology of many possible scenarios is well developed. As experimentalists, we keep an open mind.

Searches have been performed for the leptonic decays of higher mass gauge bosons both $W$-like and $Z$-like. None have been found with masses less than about $600-700 \mathrm{GeV}$. Similarly, by looking in the mass spectra of jet pairs, excited quarks can be excluded with masses less than about $600 \mathrm{GeV}$. However, such states can also influence angular distributions of lepton or quark pairs at energies well below their masses. Thus such measurements provide windows to very high masses. A recent example is the measurement[30] of lepton pair production which sets limits of 3-6 $\mathrm{TeV}$ on such compositeness scales. A few years ago, an excess of high mass events was observed[31] by the HERA experiments. One possible explanation was the existence of 1st generation leptoquarks, composite electron-quark states. Extensive measurements[32] at the Tevatron now exclude such particles unless their masses exceed about $220 \mathrm{GeV}$. Similar, if slightly weaker, limits have been place on the masses of 2 nd and 3rd generation leptoquarks.

Technicolor searches are relative newcomers to the Tevatron analysis menu. Searches have been performed[33] which provide lower limits on 

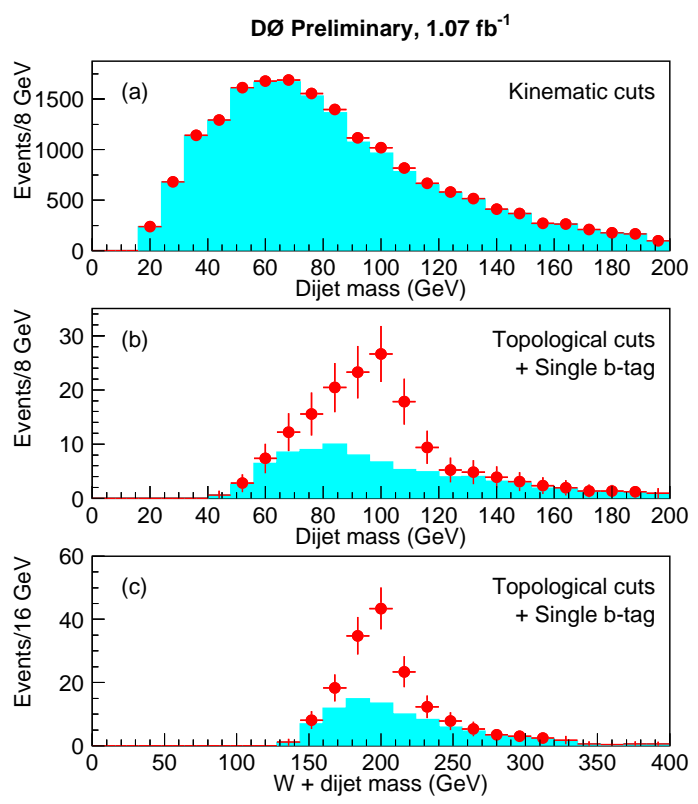

Figure 12. Mass spectra expected for technicolor signals for the $\pi_{T}$, with a mass of 110 $\mathrm{GeV}$, and the $\rho_{T}$, with a mass of $210 \mathrm{GeV}$. The points are the expected distributions including the technicolor signal; the shaded histogram is the standard model background.

the masses of technipions $\left(\pi_{T}\right)$, technirho $\left(\rho_{T}\right)$ and techniomega $\left(\omega_{T}\right)$ mesons. The techniques are illustrated by Fig. 12 in which the submass distributions of the cascade decay of a technirho $\left(\rho_{T} \rightarrow \pi_{T}+W \rightarrow b+\bar{b}+W\right)$ are plotted. With the $1 \mathrm{fb}^{-1}$ used in this study[34] the signals at 110 and 210 $\mathrm{GeV}$ are clearly visible.

TABLE 1. Mass ranges covered for a $5 \sigma$ discovery in SUSY models.

\begin{tabular}{c|c|cc} 
Model & SUSY Particle & $\begin{array}{c}\text { Run I }\left(0.1 \mathrm{fb}^{-1}\right. \\
\text { Mass Limit }(\mathrm{GeV})\end{array}$ & $\begin{array}{c}\text { Run II }\left(2.0 f b^{-1}\right. \\
\text { Mass Limit }(\mathrm{GeV})\end{array}$ \\
\hline SUGRA & & & \\
\hline & $\tilde{\chi}_{1}^{ \pm}$ & 70 & 210 \\
& $\tilde{g}$ & 270 & 390 \\
& $\tilde{t}_{1}\left(\rightarrow b \tilde{\chi}_{1}^{ \pm}\right)$ & & 170 \\
\hline GMSB & & & 265 \\
\hline & $\tilde{\chi}_{1}^{ \pm}$ & 150 & 120
\end{tabular}


The most general Supersymmetry(SUSY) theories have more than one hundred parameters and a comprehensive search is almost impossible. The usual strategy is to search for signals suggested by particular classes of models in which theoretical bias is applied to reduce the numbers of parameters to a few. Searches at the Tevatron initially concentrated on the so-called minimal-Supergravity(m-SUGRA) models by looking for multi-jet final states with missing transverse energy. The missing transverse energy is supposed to be a clear signal for the Lightest Supersymmetric Particle(LSP); if R-parity is conserved, the LSP survives the decay chain, is neutral, and will escape detection since its interactions with the matter of the detector are weak. These have been complemented by searches in channels containing leptons in the final state. The current limits[35] are in the region of squark and gluino masses of $270 \mathrm{GeV}$ if the two are approximately equal and squark masses less than about $150 \mathrm{GeV}$ are excluded for any gluino mass.

If R-parity conservation is not assumed, missing transverse energy is no longer a useful discriminant. Nevertheless searches have been performed[36, 37] particularly in channels with leptons in the final state. Limits on the squark masses in such analyses are about $260-280 \mathrm{GeV}$, and similarly for the gluino when the masses are equal.

In recent years alternative SUSY breaking scenarios to the SUGRA models have been explored. In particular, the observation[38] of a single spectacular e"e" $\gamma \gamma \mathbb{E}_{T}$ event prompted considerable activity. Theorists attempted to accomodate the event using Gauge Mediated Symmetry Breaking(GMSB) which in general leads to numerous electromagnetic objects, photons and electrons, in the final state along with missing transverse energy. Within the phase space of these models, limits on the mass of the lightest chargino, of $120 \mathrm{GeV}$ from $\mathrm{CDF}[38]$ and of $150 \mathrm{GeV}$ from $\mathrm{D} \varnothing[39]$, have been reported.

Looking to the future, a summary of what we can expect with about

$2 \mathrm{fb}^{-1}$ is displayed in Table 1 . These are the results of a fairly extensive study[40] of the potential for future SUSY exploration at the Tevatron.

\subsection{THE HIGGS}

Almost independently of theoretical religion, it is believed that some spin 0 high mass structure, known generically as the Higgs boson, must exist to generate the masses of the $W$ and $Z$ bosons. In its simplest form it could have just one observable state. Analysis of the current electroweak data suggest, as we have seen, that it be relatively light. If so it could potentially be discovered in the current running of LEP II[41], or, as concerns us here, at future running of the Tevatron. 

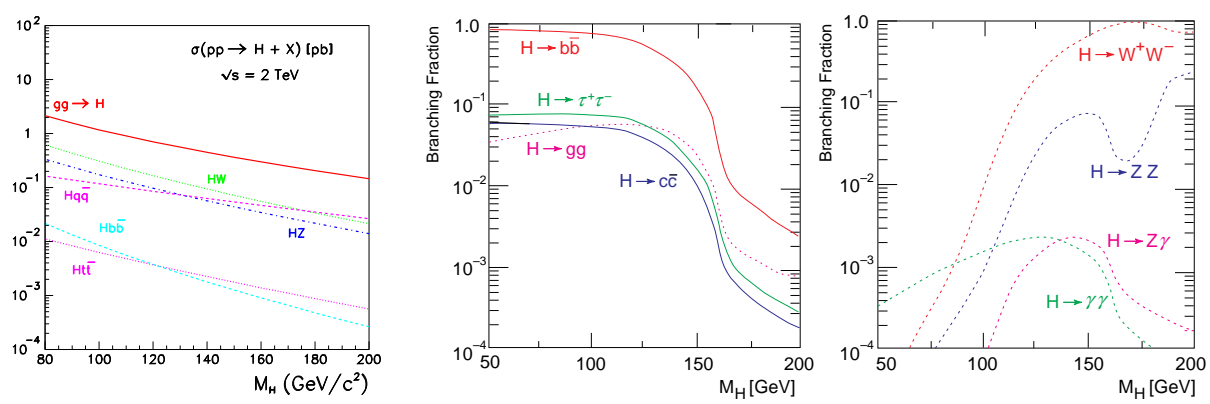

Figure 13. Higgs-boson production cross sections and branching fractions to fermions and to bosons as a function of Higgs-boson mass.

The standard model Higgs particle may be produced at the Tevatron in several different ways. As indicated in Fig. 13, the cross section for the gluon fusion process is about $1 \mathrm{pb}$ at $100 \mathrm{GeV}$. The "associated production" of an electroweak gauge boson, $W$ or $Z$, and a Higgs boson is approximately an order of magnitude less. The decay branching ratios to fermions and to bosons are also shown in the other two diagrams in Fig. 13. As expected, at low masses the $b \bar{b}$ mode dominates. However one notices that the $W W$ mode becomes large for masses in excess of $130 \mathrm{GeV}$. Folding these facts together and taking into account the need for a distinctive signature on which to trigger, and on which to key the analysis, two distinct approaches are discussed.

At low masses the approach is to look for a signal in associated production exploiting both the decays of the $W$ or $Z$ and the $b \bar{b}$ or $\gamma \gamma$ decay modes of the Higgs.

Current searches for a bosophilic Higgs use two jets[42], or two leptons[43] from the $W$ and $Z$ decays, along with two photons from the Higgs decay. $D \varnothing$ and CDF respectively place lower mass limits in the region of 80 $\mathrm{GeV}$. Using the $b \bar{b}$ mode as a signal for the Higgs, and including the $\nu \bar{\nu}$ decay among the lepton decays of the $\mathrm{Z}$, limits relevant to the standard-model Higgs are obtained[44, 43]. These do not give a mass limit but constrain the cross section at about ten times its standard model expected value. This illustrates the premium on integrated luminosity. In SUSY models the Higgs structure is more complicated and a charged Higgs exists. If its mass would be less than that of the top quark then the $H^{ \pm} b$ decay mode would compete with that of the top quark. The extent to which this would occur is controlled by the $\tan \beta$ SUSY parameter. The agreement between the observed cross section times branching ratio for the top quark into modes containing a $W$ boson and the theoretical prediction has therefore been used to place limits[45, 46] in the space of $\tan \beta$ and $m_{H}$.

With the upgraded detectors, the efficiencies are improved by a factor 


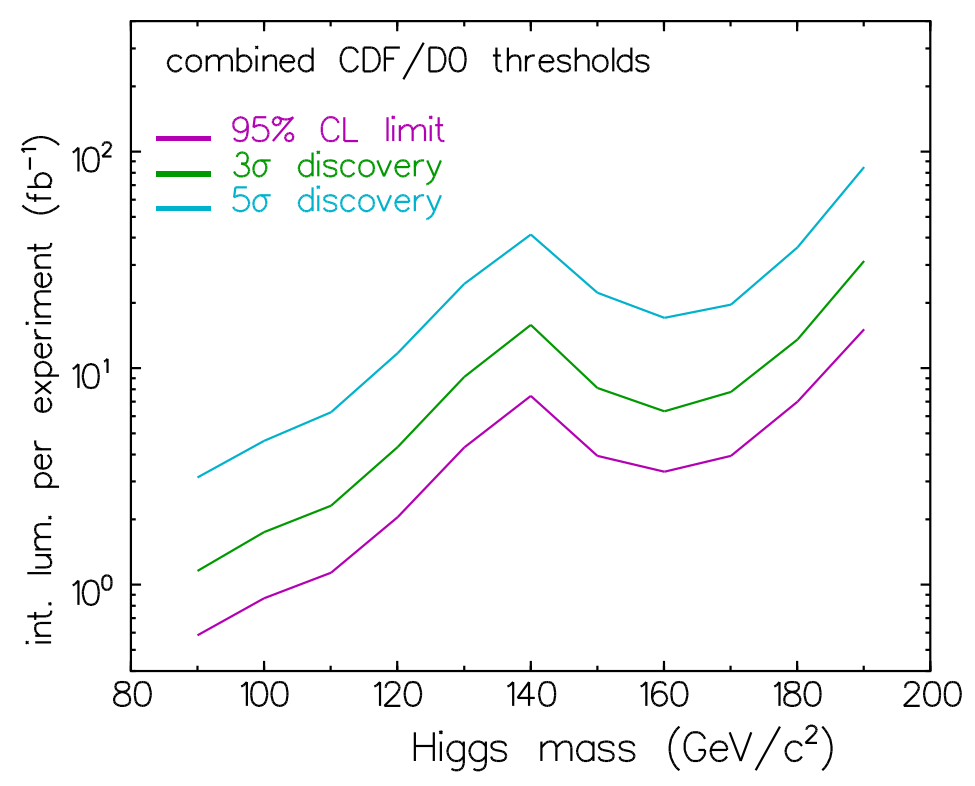

Figure 14. Luminosity required as a function of Higgs mass to achieve different levels of sensitivity to the standard-model Higgs boson.From the upper curve corresponds to a $5 \sigma$ discovery, the middle a $3 \sigma$ signal and the lower a $95 \%$ exclusion limit. These limits require two experiments, Bayesian statistics are used to combine the channels and include the improved sensitivity which would come from multivariate analysis techniques.

of about four as a result of the improved detection of $b$ quarks, and, perhaps key, the ability of each experiment to trigger on displaced vertices. Recent studies[40] take into account these improvements and improved understanding of the jet calibrations and resolutions. They find that in the relatively low mass region, below about $130 \mathrm{GeV}$, the prospects for observation of a standard model Higgs are promising. If it turns out to be possible to use all of the leptonic and haronic decay modes of $W$ and $Z$ along with just the $b \bar{b}$ decay mode for the Higgs, exclusion up to $140 \mathrm{GeV}$ and a three standard deviation hint up to about $130 \mathrm{GeV}$ could be obtained with 10 $\mathrm{fb}^{-1}$. For higher masses, the key appears to be the use[47] of the $W W^{*}$ and similar decay modes. Life is not easy when multiple modes are necessary for the observation, nevertheless, the observation of the top quark was considerably strengthened by such techniques. A $95 \%$ exclusion up to $180 \mathrm{GeV}$ might be achievable with the same $10 \mathrm{fb}^{-1}$ of integrated luminosity. A hint of a signal anywhere up to a mass of $180 \mathrm{GeV}$ probably requires $20 \mathrm{fb}^{-1}$ of integrated luminosity. This is not excluded and is a challenge which the experiments and the accelerator are keen to accept. 


\section{Conclusions}

In these lectures we have attempted to describe the Tevatron Collider complex, the experiments CDF and $\mathrm{D} \varnothing$ currently undergoing major upgrades, the physics that has come out of $100 \mathrm{pb}^{-1}$ of integrated luminosity and to

give a sense of the prospects for the future. It is safe to say that much of the physics at the Tevatron Collider has been a revelation. The experimental environment has proved to be tractable. The events are busy but the objects relevant to physics, characterised by transverse momenta in excess of $100 \mathrm{GeV}$ and of masses in the range 100 to $500 \mathrm{GeV}$, are readily observable and measurable. In particular it has proved possible to calibrate the experiments and the analyses so that precision measurements of the $W$ mass, the top quark mass and the jet cross section have been completed. Finally, complete $B$ states have been reconstructed, and their lifetimes measured and a first measurement of the $\mathrm{CP}$ violation parameter $\sin 2 \beta$ has been made. If any doubts existed a decade ago as to the breadth of the potential of the Tevatron, none should exist today.

\section{Acknowledgements}

In this electronic age we are used to having access at the push of a button to descriptions and diagrams produced in many parts of the world. This has greatly facilitated the preparation of these lectures. I would therefore like to thank my many colleagues on the CDF and DØ experiments who have helped in this work either knowingly or unknowingly. John Ellison, Paul Grannis and Nick Hadley were kind enough to read the manuscript and suggest corrections. Finally the school was immensely enjoyable and I would like to express my appreciation to the organisers, the other speakers and to the students from all of whom I learned much.

\section{References}

1. CDF Collaboration Home Page, http://www-cdf.fnal.gov/

2. DØ Collaboration Home Page, http://www-d0.fnal.gov/

3. Montgomery, H.E. (1999) Recent Results from the Tevatron Collider, Yad. Phys., Vol 62, pp. 1-9, FERMILAB-Conf-99/056-E.

4. Montgomery, H.E. (1999) Physics at the Main Injector Proceedings, DPF99 U.C.L.A. ,FERMILAB-Conf-99/057, hep-ex/9904019.

5. Montgomery, H.E. (1998) The Physics of Jets, Ecole de Gif-sur-Yvette, Centre de Physique des Particules, Marseille, France, FNAL-Conf-98/398.

6. Blazey G. \& Flaugher B. (1999) Inclusive Jet and Dijet Production at the Tevatron submitted to Ann. Rev. of Nucl. Part. Sci., hep-ex/9903058.

7. Abbott B. et al.,DØ Collaboration (1999) Inclusive Jet Cross Sections in $\bar{p} p$ Collisions at $\sqrt{s}=630 \mathrm{GeV}$ and $1800 \mathrm{GeV}$, paper submitted to the International Europhysics Conference on High Energy Physics, EPS-HEP99, 15-21 July, 1999, Tampere, Finland and to the XIX International Symposium on Lepton and Photon 
Interactions at High Energies.

8. Asakawa T. for the CDF and D $\varnothing$ Collaborations (1999) Dijet Results from CDF and DØ Proceedings of the XIII Topical Conference on Hadron Collider Physics, Tata Institute of fundamental Research, Mumbai, India, 14-20 January 1999, World Scientific, 1999.

9. Ellis S.D. and Soper D.E. (1993) Phys. Rev., D48, 3168.

10. Abbott B. et al., D $\varnothing$ Collaboration (1999) Subjet Multiplicity in Quark and Gluon Jets at D $\varnothing$, paper submitted to the International Europhysics Conference on High Energy Physics, EPS-HEP99, 15-21 July, 1999, Tampere, Finland and to the XIX International Symposium on Lepton and Photon Interactions at High Energies, hepex/9907059.

11. Abbott B. et al., DØ Collaboration (1999) to be published in Phys. Rev., FERMILAB PUB-99/171-E, hep-ex/9906025.

12. Affolder T. et al., The CDF Collaboration (1999) Submitted to Phys. Rev. Lett., FERMILAB-PUB-99/220-E; Abe F. et al., The CDF Collaboration (1999) Phys. Rev., D59, 052002.

13. Abbott B. et al, DØ Collaboration (1999) Measurement of the Transverse Momentum Distribution of $W$ and $Z$ Bosons Produced in $\bar{p} p$ Collisions at $\sqrt{s}=1.8$ $\mathrm{TeV}$,paper submitted to the International Europhysics Conference on High Energy Physics, EPS-HEP99, 15-21 July, 1999, Tampere, Finland and to the XIX International Symposium on Lepton and Photon Interactions at High Energies, hepex/9907044.

14. Abe F. et al., The CDF Collaboration (1998) Phys. Rev. Lett., 81, 1367.

15. Nakada T. (1999) Lectures at this School

16. Buras A. (1996) TUM-HEP-255-96, Sep 1996, hep-ph/9609324.

17. Abbott B. et al., D $\varnothing$ Collaboration (1999) Forward Rapidity Muon and $b$-quark Production in $p \bar{p}$ Collisions at $\sqrt{s}=1.8 \mathrm{TeV}$, Paper \#453 submitted to the XXIX International Conference on High Energy Physics - ICHEP98, July 23-29, 1998, Vancouver, B.C., Canada.

18. Abe F. et al., CDF Collaboration (1998) Phys. Rev. Lett., 81, 5513.

19. Gershtein S.S., Kisilev V.V., Likhoded A.K., Tkabladze A.V. (1995) Uspeki Phys. Nauk., 165, pp. 3-40., IHEP 94-81, 1994.

20. Abe F. et al., The CDF Collaboration (1998) Phys. Rev. Lett, 82, 3576; Paulini M. (1999) Int. J. Mod. Phys., A14, 2791.

21. Affolder T. et al., The CDF Collaboration (1999) submitted to Phys. Rev. D, FERMILAB-PUB-99/225-E.

22. Affolder T. et al., The CDF Collaboration (1999) Submitted to Phys. Rev. Lett. FERMILAB-PUB-99/257-E.

23. Chakraborty Dhiman for the CDF \& D $\varnothing$ Collaborations (1999) Study of the Top Quark at the Tevatron, Proceedings of the XIII Topical Conference on Hadron Collider Physics, Tata Institute of fundamental Research, Mumbai, India, 14-20 January 1999, World Scientific, 1999.

24. Ellison J. and Wudka J. (1998) Study of Trilinear Gauge Boson Couplings at the Tevatron Collider Ann. Rev. Nucl. Part. Sci., 48, 33.

25. Rimondi F. CDF and D $\varnothing$ Collaborations (1999) $W \rightarrow \tau \nu$ and $\Gamma_{W}$ Studies at the Tevatron Collider Proceedings of the XIII Topical Conference on Hadron Collider Physics, Tata Institute of fundamental Research, Mumbai, India, 14-20 January 1999, World Scientific, 1999.

26. Lancaster M. (1998) Proceedings of Results and Perspectives in Particle Physics, La Thuile, Italy.

27. Abbott B. et al., DØ Collaboration (1999) to be published in Phys. Rev. Lett, FERMILAB PUB-99/259-E, hep-ex/9909030 ; submitted to Phys. Rev. D., hepex/9908057, FERMILAB-Pub-99/237-E).

28. LEP Electroweak Working Group (1999) http://www.cern.ch/LEPEWWG/plots/summer99/ 
29. Sliwa K. (1999) Top Mass and Cross Section Results from CDF and DØ at the Fermilab Tevatron Proceedings of the XIII Topical Conference on Hadron Collider Physics, Tata Institute of fundamental Research, Mumbai, India, 14-20 January 1999, World Scientific, 1999.

30. Abbott B. et al., DØ Collaboration (1999) Phys. Rev. Lett., 82, 4769.

31. Cashmore R. (1999) Lectures at this School

32. Hagopian S. for the DØ and CDF Collaborations (1999) Leptoquark Summary from the Tevatron Proceedings of the XIII Topical Conference on Hadron Collider Physics, Tata Institute of fundamental Research, Mumbai, India, 14-20 January 1999, World Scientific, 1999.

33. Abe F. et al., The CDF Collaboration (1999) Phys. Rev. Lett., 83, 3124; Affolder T. et al., The CDF Collaboration, (1999) submitted to Phys. Rev. Lett., FERMILABPUB-99/141-E. ; Abe F.et al., The CDF Collaboration (1998) submitted to Phys. Rev. Lett., FERMILAB-PUB-98/321-E.

34. Eichten E., Lane K., Womersley J., (1997) Phys. Lett., B405, 305.

35. Pagliarone C. (1999) SUSY Searches at Tevatron Collider Proceedings of the XIII Topical Conference on Hadron Collider Physics, Tata Institute of fundamental Research, Mumbai, India, 14-20 January 1999, World Scientific, 1999.

36. Abe F. et al., The CDF Collaboration (1999) Phys. Rev. Lett., 83, 2133; Abe F. et al., The CDF Collaboration (1998) submitted to Phys. Rev. Lett., FERMILABPUB-98/374-E.

37. Abbott B. et al., DØ Collaboration (1999) Search for R-parity Violating Supersymmetry in the Dielectron Channel, to be published in Phys. Rev. Lett., hepex/9907019.

38. Abe F. et al., The CDF Collaboration (1998) Phys. Rev. Lett., 81, 1791; Abe F. et al., The CDF Collaboration, (1999) Phys. Rev., D59, 092002.

39. Abbott B. et al., DØ Collaboration (1997) Phys. Rev. Lett., 78, 2070; Abbott B. et al., DØ Collaboration (1999) Phys. Rev. Lett., 82, 29.

40. Physics at Run II - Supersymmetry/Higgs, http://fnth37.fnal.gov/susy.html

41. Fernandez H., (1999) Lectures at this School.

42. Abbott B. et al., 1999, DØ Collaboration (1999) Phys. Rev Lett., 82, 2244.

43. Wu X. for the CDF and D $\varnothing$ Collaborations (1999) Higgs Search at the Tevatron Proceedings of the XIII Topical Conference on Hadron Collider Physics, Tata Institute of fundamental Research, Mumbai, India, 14-20 January 1999, World Scientific, 1999.

44. Abe F. et al., The CDF Collaboration (1998) Phys. Rev. Lett., 81, 5748.; Abe F. et al., The CDF Collaboration (1998) Submitted to Phys. Rev. Lett., FERMILABPUB-98/252-E.

45. Abe F. et al., The CDF Collaboration (1997) Phys. Rev Lett., 79, 357.

46. Abbott B. et al., DØ Collaboration (1999) Phys. Rev. Lett., 82, 4975.

47. Han T, Turcot A. and Zhang R. (1999) Phys.Rev. D59 093001. 